\title{
Fixed perfusion defects revisited
}

\author{
Ami E. Iskandrian, MD, MASNC ${ }^{\mathrm{a}}$ \\ a UAB, Cardiology, Birmingham
}

Received Dec 10, 2020; accepted Dec 10, 2020

doi: $10.1007 / \mathrm{s} 12350-020-02505-6$

In this issue of the Journal, there are 2 invitational papers, one by Gewirtz and the second by Dilsizian on fixed perfusion defects (PD). This topic is important and affects directly patient management.

The fixed PD under consideration does not include and should not include attenuation artifacts as those may look like fixed PD but the myocardium is normal otherwise. The term fixed PD implies the presence of a PD that appears of same size, location and severity on post- stress and rest images. This term started with planar myocardial perfusion imaging and was subsequently adopted by SPECT and PET.

We know from the work of both above mentioned authors and others that fixed PD may appear reversible with some interventions (like thallium reinjection or after nitroglycerine or a more powerful stress) and some improve after coronary revascularization. We know also that some Fixed PDs are truly fixed; for example, after percutaneous coronary interventions in patients with STelevation myocardial infarction, where the culprit artery is rendered patent, but myocardial necrosis had occurred, analogous to closing the barn door after the horse had left the barn! I like to refer to such PD as true positives! These fixed PD are easy to understand from a mechanistic viewpoint.

On the other hand, fixed PDs in territories subtended by "severely" stenosed coronary arteries are not intuitively easy to understand as the question is why don't we see ischemia in the outer layer of the myocardium (non-infarcted myocardium)? I like to refer to such PDs as false positives!

How then to differentiate the true positives from the false positives? I hope the 2 papers help the readers in re-thinking of this issue every time they report fixed PD.

Alternative terms used to describe fixed PD include scar, non-reversible defects and non- viable myocardium. These terms are like 2 people describing the 2 ends of an elephant, true but incomplete.

Our reports need to be precise and user-friendly that help rather than hinder patient management. We need to keep in mind that our reports are unlikely read by imaging specialists or those who attend national or international imaging meetings or webinars who are familiar with our jargons. I submit therefore that even the term PD may not be ideal. Scar is much easier to understand as it is a universal term, but it should be modified such as partial thickness scar or more conventionally as mild, moderate or severe (in addition to its [size small, medium or large] and location). These are better understood by referring physicians than summed rest score (which says nothing about location, size or severity)!

Finally, with the same day rest-stress protocol using Tc-labeled tracers and three times higher dose at stress than rest, the shine through phenomena could result in under-estimation of reversibility (over-estimation of scar).

It is amazing that after five decades, we come back to the basics of what seemed obvious. But self- examination also means that our field has matured, and we are now zooming on critical parts of what we say and write for the betterment of our patient care.

\section{Disclosures}

There is nothing to disclose.

Publisher's Note Springer Nature remains neutral with regard to jurisdictional claims in published maps and institutional affiliations. 